\title{
INSPIRE: Interplanetary NanoSpacecraft Pathfinder In Relevant Environment
}

\author{
Andrew Klesh ${ }^{1}$, John Baker, Julie Castillo-Rogez, Lauren Halatek, Neil Murphy, Carol \\ Raymond \\ Jet Propulsion Laboratory, California Institute of Technology, Pasadena, CA 91105 \\ John Bellardo \\ California Polytechnic State University, San Luis Obispo, CA 93407 \\ James Cutler \\ University of Michigan - Ann Arbor, Ann Arbor, MI 48109-2140 \\ and \\ Glenn Lightsey \\ University of Texas - Austin, Austin, Texas 78712-1221
}

\begin{abstract}
The INSPIRE project would demonstrate the revolutionary capability of deep space CubeSats by placing two nanospacecraft in Earth-escape orbit. Prior to any inclusion on larger planetary missions, CubeSats must demonstrate that they can operate, communicate, and be navigated far from Earth - these are the primary objectives of INSPIRE. Spacecraft components, such as a JPL X-band radio and a robust watchdog system, would provide the basis for future high-capability, lower-cost-risk missions beyond Earth. These components should enable future supplemental science and educational opportunities at many destinations.

The nominal INSPIRE mission would last for three months and achieve an expected Earth-probe distance of $1.5 \times 10^{8} \mathrm{~km}$ (dependent upon escape velocity as neither spacecraft will have propulsion capability). The project would monitor onboard telemetry; operate, communicate, and navigate with both spacecraft; demonstrate cross-link communications; and demonstrate science utility with an onboard magnetometer and imager. Lessons learned from this pathfinder mission should help to inform future interplanetary NanoSpacecraft and larger missions that might use NanoSpacecraft components.
\end{abstract}

This paper provides an update on the INSPIRE development effort, and for each subsystem, provides lessonslearned for future inte ${ }^{1}$ rplanetary Nanospacecraft efforts.

\section{Introduction}

$\mathrm{D}$ ue to be deep-space-flight-ready in summer 2014, the Interplanetary NanoSpacecraft Pathfinder In Relevant

Environment (INSPIRE) mission would become the world's first dedicated interplanetary CubeSat, opening deepspace heliophysics and planetary science to the CubeSat revolution. The INSPIRE project intends to accomplish a tiered set of technology-demonstration and education objectives: it will demonstrate that NASA CubeSats can (1) operate, communicate, and be navigated far from Earth; (2) host and support CubeSat components whose hardware has not yet had the opportunity to fly in deep space; and (3) deliver useful science that in turn opens future mission opportunities for investigators. These objectives intimately embody many NASA strategic objectives, and would enable a new generation of low-cost solar system explorer.

1 Principal Investigator, INSPIRE Project, 4800 Oak Grove Blvd, Pasadena, CA 91109, andrew.t.klesh@jpl.nasa.gov

American Institute of Aeronautics and Astronautics 
The INSPIRE flight system comprises two identical, three-axis-stabilized $3 \mathrm{U}$ spacecraft that combine existing subsystems for C\&DH watchdog, attitude determination, and power functions with next-step modifications of subsystems for cold-gas attitude control and deep-space navigation \& communication. Demonstrating the integrated system performance of these core spacecraft compon ${ }^{2}$ ents will establish a proven foundation for diverse future NASA missions to host special-purpose payloads in deep space. The spacecraft would also host a science payload: a half-U JPL compact vector-helium magnetometer to measure the fine structure of the solar wind, and prove the science utility of such a small platform. The project significantly leverages experience from prior CLI launch opportunities, including hardware, software, and personnel from the RAX2, M-Cubed/COVE, GRIFEX, CP-series, BEVO-2, and ArmadilloSat CubeSat projects.

INSPIRE's demonstration of CubeSat functionality and utility in the deep-space environment would be a crucial stepping-stone for interplanetary CubeSats. By leveraging JPL's 50-plus years of deep space experience, INSPIRE should establish a flight heritage for future interplanetary CubeSat missions, as well as creating a cadre of partner universities experienced with the challenges of interplanetary missions.

In this paper we will provide an update to the technical details of the INSPIRE project, discuss current status and lessons learned to date, and detail some of the future missions enabled by the INSPIRE project. Additional details can be found in Reference 1 .

\section{Project Technical Design}

The INSPIRE mission consists of two identical spacecraft, launched simultaneously as secondary payloads, and deployed to Earth-escape. The nominal mission would last for three months and achieve an expected Earth-probe distance of $1.5 \times 10^{8} \mathrm{~km}$ (dependent upon escape velocity as neither spacecraft would have propulsion capability). Two spacecraft provide redundancy to reduce the risk in meeting the project goals (with the exception of crosslink communications, which will be demonstrated early in the mission timeline).

\section{A. Command and Data Handling}

The core of the INSPIRE spacecraft consists of two parts: the watchdog board and the Iris deep space transceiver/transponder. The watchdog board is based on the proven MSP430 microprocessor flown on many successful CubeSat missions, including RAX-2 and Delfi-C3, and has also demonstrated limited radiation tolerance in experimental testing (Ref 1). The board will be responsible for basic command and data-handling; interfacing with the radio, attitude control system, star tracker, electrical power system, and payloads via UART, $\mathrm{i}^{2} \mathrm{c}$ and SPI lines; and monitoring spacecraft health. It also provides a cascading watchdog architecture responsible for monitoring and resetting all subsystems, if necessary, in the event of SEUs or other fault conditions. The C\&DH system contains two-SD cards (one serves as a cold-spare), a real-time-clock, onboard UHF radio, MEMs gyro, watchdog circuitry, and even a hardwired identification circuit to uniquely identify the spacecraft. Heritage from the C\&DH systems is derived directly from RAX and RAX-2 spacecraft, where this functionality was separated into three boards. AstroDev, Inc has developed a new generation system, with the same circuitry, that fits within a single board.

As is usual in the development of CubeSat missions, interfacing between systems remains the primary challenge in development. Since the Command and Data Handling system is at the center of the spacecraft, it is often here that challenges are found, including current-leaks through I/O lines, isolation issues for powering boards, and related fault-tolerance problems. Depending on the development schedule, being able to make late changes to this board can significantly simplify changes to the system - centralizing changes to interoperability rather than having to change many boards throughout the spacecraft.

Though the MSP430 is capable, it's purpose is solely for minimum-spacecraft functionality (and INSPIRE would be designed for minimum capability) - a secondary processor able to perform more complex operations should be available for payload operations.

\section{B. Communication}


Primary communication with the spacecraft would be over the DSN compatible Iris X-Band transceiver/transponder. The radio relies on dual receiver / transmitter antennas located on either end of the spacecraft, providing nearly omnidirectional communication coverage. With a $1 \mathrm{~W}$ transmitter, the link budget shows that the radio can communicate at greater than $1 \mathrm{kbps}$ at $1.5 \mathrm{M} \mathrm{km}$, but the performance is flexible for data rates between $62.5 \mathrm{bps}$ up to $256 \mathrm{kbps}$ (depending on range). The radio provides coherent uplink and downlink allowing for accurate ranging and Doppler measurements to be made on the ground. Away from a gravitational body, the expected navigational accuracy is less than $500 \mathrm{~km}$ relatively close to Earth, and between 1000 and $2000 \mathrm{~km}$ further along the mission timeline.

The CCSDS compliant radios have a limited amount of onboard memory to buffer received and transmitted data, and also allow for a so-called "firecode" providing the ability to externally reset the spacecraft if needed. The radios themselves are quite robust, and build upon lessons learned from the from the M-Cubed/COVE processing experiment for digital board design. The Iris radio itself builds from the Electra radios at Mars and the Low-Mass Radio Science Transponder (LMRST) system slated to fly on the LMRST-Sat CubeSat project.

In addition to the X-band radio, an AstroDev Lithium UHF radio is built onto the C\&DH board to provide cross-link capability between the spacecraft. This allows for direct-relay and store-and-forward-relay communications between the ground and either spacecraft. The UHF radios also allow for a fire-code command to provide additional reset capability.

To support the CCSDS compatible radios, INSPIRE would make use of the NASA AMMOS (Advanced MultiMission Operations System) software. The software is CCSDS and DSN compatible, provides both uplink and downlink capability, and has been proven in many previous missions, including MSL and the upcoming SMAP mission. Adopting a standard software would provide significant reliability to the INSPIRE system, and lower overall project risk. The software also has a flexible database and framework backing, providing for scripting capabilities for new tools, and easy integration into the INSPIRE project.

As a low-cost mission with a small team, the INSPIRE project would also use the AMMOS software during the integration and test phase to both exercise the spacecraft functionality with flight-like tools, as well as train the engineers for later flight operations (likely the same team for integration and test will fly the spacecraft). This increases overall training time with the actual flight hardware prior to flight, and helps to develop the needed tools, dictionaries, and procedures for flight ops.

\section{Attitude Determination and Control}

A Blue Canyon Technologies commercial star tracker / imager will provide a majority of the attitude control information, including quaternions and rates, but chip-scale gyros and photodiodes will provide verification. Lowfidelity attitude estimation algorithms will verify sun-direction (the most critical angle to acquire) to assure correct panel orientation and keep-out angles for the exposed devices.

Attitude control would be provided by a four-thruster cold-gas system provided by U. Texas-Austin. Building from MEPSI heritage from STS-116, the tank is 3D-printed, and contains thrusters that can be fired together or independently depending on direction and impulse required. The C\&DH system would run a limited attitude control algorithm for both detumbling and nominal flight operations, that will take into account relevant sensors and spin rates - for instance, during initial detumble, only the photodiodes and gyros will be used to mitigate expected motion blur of the star tracker during the initial fast rotatations. The 3D-printed tank provides design flexibility and growth of system components until late in the design process. 


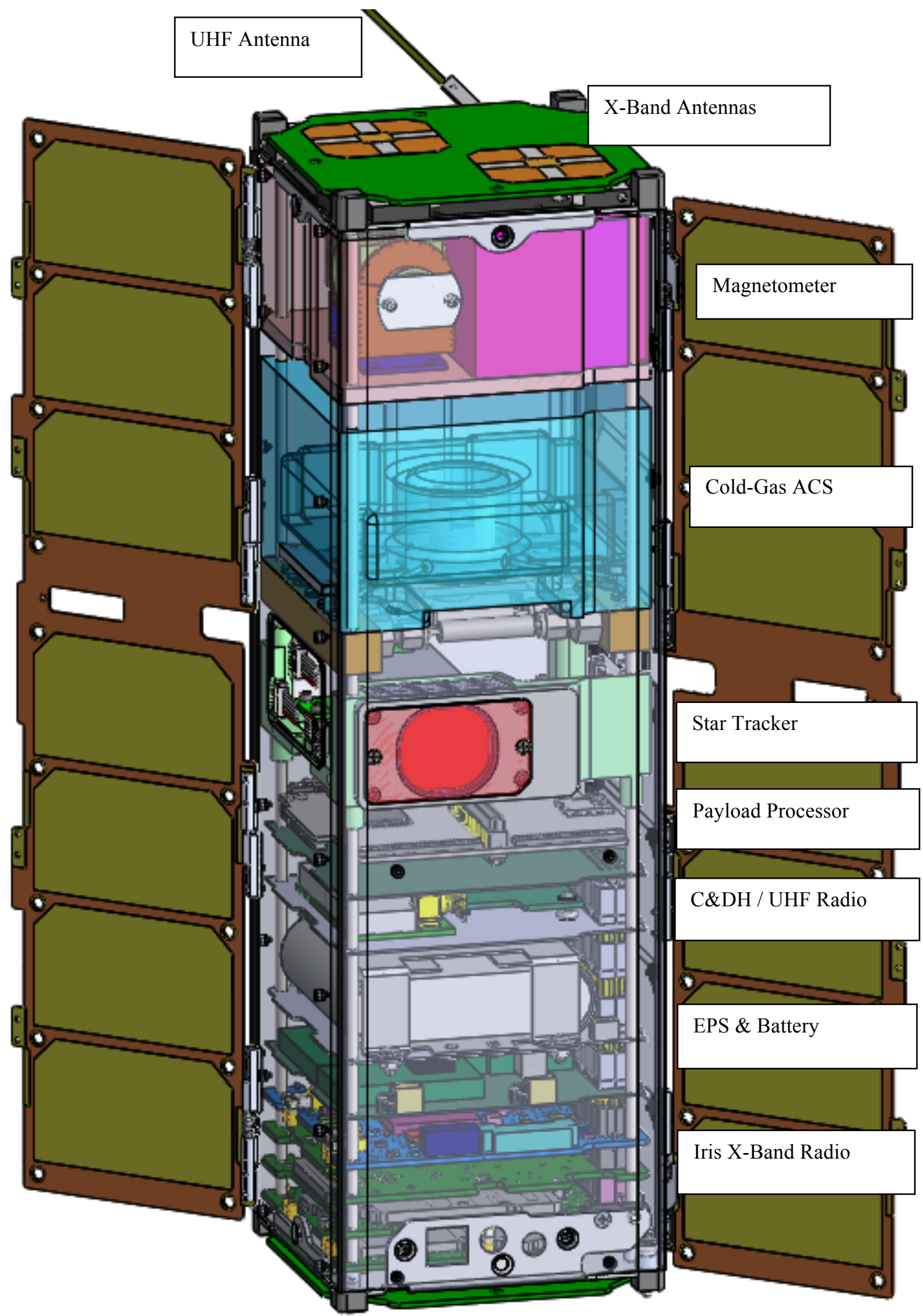

Figure 1 - Transparent view of one of the identical proposed INSPIRE spacecraft 
One challenge of a CubeSat system is the highly integrated (and small) nature of the system. As a secondary payload, it is often the case that this small system may also be sitting on the shelf for several months before flight, with limited (through the P-POD) access to the spacecraft. As an ACS system has potential for leaks, the INSPIRE project would require a fill port external to the spacecraft to fill the tank once it is integrated, yet fit within the confines of the P-POD. This challenge has previously arisen during ACS tank testing in a thermal-vac chamber (especially as the low-mass of the tank can cause flash-heating of the refrigerant upon the manifold), yet can be solved with standard filling procedures and mechanisms.

Likewise, a cold-gas system located near imagers can cause outgassing problems, and should be mitigated prior to the final design.

\section{Electrical Power System}

The power system is built from RAX/RAX-2 heritage, which has now cumulatively demonstrated over two years of successful operations in Earth-orbit. Fixed solar panels cover three sides of the INSPIRE spacecraft, and together with two deployable panels, would provide greater than $20 \mathrm{~W}$ of onboard power when oriented toward the sun.

As INSPIRE would be a technology demonstration mission, capability margin would be provided to assure mission success - thus, even if the deployable panels do not extend, enough power would be available to charge the batteries and perform mission operations, albeit with a change in duty cycling.

\section{E. Integration and Testing}

JPL is designing and integrating the spacecraft while the university partners are providing many of the subsystems: U. Texas has provided the attitude control system, U. Michigan / AstroDev has provided the electrical power system and C\&DH, as well as serving as a groundstation, CalPoly has provided a processing board, and GAVRT is providing receive-only operations at DSS-13.

Each of the university subsystems is being built with JPL oversight and consulting, thus providing the CubeSat community several experienced deep space developers for future interplanetary missions. These subsystems will be integrated at JPL in collaboration with the students who developed them through internship programs. Each spacecraft will be subject to extended bench-top operations to verify functionality. The spacecraft will then be tested as a unit prior to launch delivery, undergoing thermal/vac, vibe and shock testing.

The value added by students integrating components of the spacecraft while at JPL is of great benefit. Many systems are able to be improved through discussion and alteration by experts, and changes can likewise be made as the full integrated system is considered, rather than only a subsystem. Each student is also able to work with other subsystems, gaining a better understanding of the overall vehicle, and able to make recommendations at the system level.

\section{F. Payloads}

INSPIRE would carry two payloads: a compact vector-helium magnetometer, and a COTS payload processor with imager. Each of these boards extends the functionality of the spacecraft toward future endeavors.

\section{Compact Vector-Helium Magnetometer}

The JPL / UCLA compact vector-helium magnetometer (CVHM) builds from extensive magnetometer development, from Mariner, through Cassini, while simultaneously significantly reducing overall size: the entire instrument takes up approximately $5 \times 10 \times 10 \mathrm{~cm}$ and $0.5 \mathrm{~kg}$, including deployable boom. With a stability of less than $10 \mathrm{pT}$, the CVHM provides a significant improvement on previously flown CubeSat magnetometers. As the spacecraft separate from each other after deployment from the P-POD, the magnetometer measurements would provide fine-grain, high fidelity measurements of the solar wind, providing a new look into turbulence effects. Depending upon trajectory (expected Earth-leading or Earth-trailing), the magnetometers may also provide a real-time gradient look across the bow-shock of Earth's magnetosphere during the mission.

The small size of the magnetometer makes it ideal for use on both large and small missions in the future, while remaining "science-grade". Extensive work is being carried out to enable accurate measurements within this small formfactor. 


\section{High Powered Payload Processor}

The CalPoly / Tyvak processing board would also fly on the INSPIRE spacecraft, with software derived from the IPEX CubeSat mission. This agile-science software should allow for advanced processing of images and magnetometer data to streamline downlink and increase science return. The processing system (otherwise known as the Intrepid system board), will also support a small imager, with a goal of taking images of the other spacecraft, the Earth and the Moon. This processor would be monitored by the primary INSPIRE systems and reset as needed to mitigate latch-up conditions. The high powered processor provides capabilities for onboard science data processing, backup C\&DH functionality (though not implemented on INSPIRE), and additional telemetry sensors to monitor the spacecraft

\section{Conclusion}

Once proven in flight, interplanetary CubeSats could provide multiple adjunct roles in support of deep-space human missions, e.g., external inspection of flight systems, "third viewpoint" observation of operations, advance reconnoitering of exploration sites, and continuous monitoring observations after humans depart a site. CubeSats are widely recognized as a disruptive technology. Yet programmatic missions on which their deep space development could piggyback are increasingly rare; and competitively selected PI-led missions are unlikely to incorporate CubeSat-platform based operations concepts until such platforms are demonstrated, yielding quantified heritage performance.

While non-validated technology continues to be a major hurdle for mission development, the establishment and continued progress of the INSPIRE concept has enabled other interplanetary missions to be proposed and funded from a number of institutions. This growing confidence in "low-cost / high-risk" missions built from CubeSat technology has both emboldened proposers, and highlighted technological hurdles still needing to be solved. Some of these hurdles include high-rate communications at large distances, pointing accuracy and stability, and a propulsion system for different regimes of flight - high-thrust, short duration; low-thrust, long duration; and escape regimes, where both efficiency and total delta- $\mathrm{V}$ matter.

A successful launch of the INSPIRE spacecraft would enable supplemental science and education opportunities for CubeSats in the next round of interplanetary proposals, including Discovery, New Frontiers and Explorer, all expected in the 2015/2016 timeframe. While a 2014 launch would provide enough opportunity that lessons learned from the several month mission could be incorporated into the larger mission proposals in following years, continued progress on INSPIRE would also help build confidence in the components of the spacecraft that might be useful in other proposal and instrument opportunities.

INSPIRE would provide the first spacecraft in a new generation of explorers, and takes advantage of extensive collaborations between the CubeSat community and experienced solar-system explorers. This exciting mission should enable novel science, demonstrate capable components, and kick-start the journey of CubeSats beyond lowEarth orbit.

\section{Acknowledgments}

This work has been carried out at the Jet Propulsion Laboratory, California Institute of Technology, under contract to NASA. Government sponsorship acknowledged.

The authors of this work are only a small part of the INSPIRE project team, and the project would not be accomplished without them. The authors would like to acknowledge their dedication and innovation in carrying out this project, especially the efforts of Devin Hupp, Travis Imken, So-Hee Kang, and Matt Zimmerer, the INSPIRE 2013 summer interns.

\section{References}

${ }^{1}$ Klesh, A., et al. "INSPIRE: Interplanetary NanoSpacecraft Pathfinder In Relevant Environment", $27^{\text {th }}$ AIAA Small Satellite Conference, 2013.

${ }^{2}$ Vladimirova, T., et al. "Characterising Wireless Sensor Motes for Space Applications", Second NASA/ESA Conference on Adaptive Hardware and Systems, 2007. 